\title{
Spektral Eşleştirme Yöntemleri Kullanarak Hiperspektral Görüntülerin Seyrek Gösterim Tabanlı Sınıflandırılması
}

\author{
Tuğcan DÜNDAR ${ }^{1 *}$, Taner İNCE ${ }^{2}$ \\ ${ }^{1}$ Gaziantep Üniversitesi, Mühendislik Fakültesi, Elektrik ve Elektronik Mühendisliği Bölümü, Gaziantep \\ (dundar@gantep.edu.tr) ORCID ID 0000 - 0003 - 1374-8651 \\ ${ }^{2}$ Gaziantep Üniversitesi, Mühendislik Fakültesi, Elektrik ve Elektronik Mühendisliği Bölümü, Gaziantep \\ (tanerince@gantep.edu.tr) ORCID ID 0000 - 0003 - 1757-5209
}

\section{$\ddot{O} z$}

Seyrek gösterim tabanlı yaklaşımlar sinyal ve görüntü işleme alanlarında gösterdikleri performanstan dolayı son zamanlarda hiperspektral görüntüler üzerine de uygulanmaya başlanmış ve başarılı sonuçlar sağlanmıştır. Hiperspektal görüntü içerisindeki uzamsal bilginin de sınıflandırma işlemine dahil edilebilmesi için ortak seyrek gösterim sınıflandırıcı (OSGS) modeli geliştirilmiştir. Fakat bu modelde test pikseli etrafındaki sabit boyutlu bir pencere içerisindeki tüm komşu piksellerin ağırlık oranlarının eşit olduğu varsayılmaktadır. Özellikle de pencere boyutu arttıkça farklı sınıfa ait piksellerin sınıflandırma işlemine dahil olacağı düşünülürse hata payı artacaktır. Bu soruna bir çözüm üretebilmek için pencere içerisindeki merkez test pikseli ve her bir komşu piksele 3 adet spektral eşleştirme yöntemi uygulayıp OSGS ile birleştiren 3SE-OSGS metodu önerilmiştir. Eşleştirme yöntemlerinden elde edilen verilere ve eşik değerine göre ilgili komşu pikselin seçilmesi veya seçilmemesi sağlanmıştır.

Anahtar Kelimeler: seyrek gösterim, spektral eşleştirme, hiperspektral görüntü, sinıflandırma.

\section{Sparse Representation-Based Classification of Hyperspectral Images Using Spectral Matching Methods}

\begin{abstract}
Sparse representation based approaches have recently begun to be applied to hyperspectral images due to the performance displayed in areas such as signal and image processing and successfull results have been achieved. The joint sparse representation classifier (JSRC) model has been developed so that spatial information in the hyperspectral image can be included in the classification process. However, it is assumed that the weight ratios of all neighboring pixels in a fixed size window around the test pixel are equal in this model. Particularly, as the window size increases, the error rate will increase if it is considered that the pixels belonging to different classes will be included in the classification process. In order to solve this problem, 3SM-JSRC method utilizing 3 spectral matching methods to central test pixel and each neighbor pixel in the window and combines with JSRC is proposed. It is provided that the neighboring pixel is selected or not selected according to the data obtained from the matching methods and the threshold value.
\end{abstract}

Keywords: sparse representation, spectral matching, hyperspectral image, classification.

\footnotetext{
* Sorumlu Yazar
} 


\section{GÍRİ̧}

Hiperspektral görüntüler birbirine bitişik olarak konumlandırılmış çok sayıda spektral banttan oluşmaktadır (Christophe, Leger ve Mailhes, 2005). Görüntü içerisindeki spektral bilginin oldukça fazla olması yeryüzündeki materyallerin tespitine ve siniflandirılmasina olanak sağlamaktadır. Bu sebeple de askeri, tarım ve mineraloji gibi uygulama alanlarında sıklıkla kullanılmaktadır (Datt, McVicar, Niel, Jupp ve Pearlman, 2003; Garcia ve Ustin, 2001; Manolakis ve Shaw, 2002; Shukla ve Kot, 2016). Hiperspektral görüntü sınıflandırma işlemindeki amaç verilen bir test pikselini sınıflandırma metotları yardımı ile ait olduğu doğru sınıfa atamaktır. Bu amaçla önerilen ilk çalışmalarda hiperspektral görüntüdeki spektral bilgi kullanılarak destek vektör makinaları tabanlı metotlar (Bruzzone, Chi ve Marconcini, 2006; Chi ve Bruzzone, 2007; Melgani ve Bruzzone, 2004), rastgele ormanlar (Dalponte, Ørka, Gobakken, Gianelle ve Næsset, 2013; Ham, Chen, Crawford ve Ghosh, 2005) ve k en yakın komşuluk (Ma, Crawford ve Tian, 2010), yapay sinir ağları (Ratle, Camps-Valls ve Weston, 2010; Zhong ve Zhang, 2012), lojistik regresyon (J. Li, Bioucas-Dias ve Plaza, 2012, 2013), genetik algoritma (Stavrakoudis, Galidaki, Gitas ve Theocharis, 2012) ve karar ağaçları (Goel vd., 2003) gibi yöntemler sayesinde sinıflandırma işlemi yapılmıştır.

Son zamanlarda, seyrek gösterim tabanlı siniflandirma modelleri hiperspektral görüntüler için önerilmiştir. Chen vd. tarafından spektral bilgiyi kullanan seyrek gösterim sinıflandırıcısı (Sparse Representation Classifier - SRC) (Chen, Nasrabadi ve Tran, 2011) önerilmiştir. SRC modelinde bir hiperspektral test pikselinin tüm sinıflara ait eğitim örneklerinden oluşan eğitim sözlüğü içerisinden sadece birkaç eğitim örneği ile temsil edilebileceği önerilmektedir. Ayrıca, görüntü içerisindeki uzamsal bilginin yani piksellerin komşuluk bilgilerinin sınıflandırma işlemine dahil edildiği ortak seyrek gösterim sınıflandırıcı (Joint Sparse Representation Classifier JSRC) (Chen vd., 2011) modeli de yine Chen vd. tarafından aynı çalışmada sunulmuştur. JSRC modelinde pencere içerisindeki merkez test pikseli etrafındaki piksellerin hepsinin de aynı sınıfa ait olduğu varsayılır ve sınıflandırma işlemi yapılır.

Sonraki yıllarda JSRC yönteminin sağladığ1 sinıflandırma doğruluk sonuçlarında artış sağlayabilmek için birçok çalışma yapılmıştır (Dundar ve Ince, 2019; Fang, Li, Kang ve Benediktsson, 2014, 2015; W. Li ve Du, 2014; Sun, Qu, Nasrabadi ve Tran, 2014; Tu vd., 2018). (Tu vd., 2018)'de önerilen CCJSR (Correlation Coefficient - Joint Sparse Representation) çalışmasında test pikselinin sınıfını belirlerken hesaplanan artık değere test pikseli ile eğitim örnekleri arasındaki benzerlik korelasyon katsayısı yardımı ile eklenmiştir. (Fang vd., 2014)'daki MASR (Multiscale Adaptive Sparse Representation) metodunda test pikseli etrafinda tek bir pencere oluşturmak yerine farklı boyutlarda pencereler oluşturularak uzamsal bilginin daha fazla kullanılması amaçlanmıştır. (Fang vd., 2015)'da önerilen SBDSM (SuperpixelBased Discriminative Sparse Model) yöntemi sayesinde sabit boyutlu pencere yerine uzamsal bilgiye göre şekil ve boyut değiştiren süperpikseller test pikseli etrafinda oluşturulmuştur ve uzamsal bilgi daha verimli kullanılmıştır. Test örneği etrafında tek bir süperpiksel yerine farklı ölçeklerde süperpikseller oluşturan ve sinıflandırma sonuçlarına rehberli filtreyi ekleyerek uzamsal bilginin daha da verimli kullanılmasını sağlayan yöntem MSS-GF (Multi Scale Superpixels-Guided Filter) ise (Dundar ve Ince, 2019)'de önerilmiştir. (Sun vd., 2014)'de ise siniflandırma problemine bazı seyreklik temelli sınırlandırmalar getirilerek sınıflandırma sonuçlarında artış sağlanması amaçlanmıştır. (W. Li ve $\mathrm{Du}, 2014$ )'de yapılan çalışmada komşuluk bilgilerini ve test pikseli ile eğitim örnekleri arasındaki benzerlikleri sınıflandırma yöntemine dahil eden JCR (Joint Collaborative Representation) metodu önerilmiştir.

JSRC yönteminde özellikle de pencere boyutu arttıkça farklı sinıflara ait pikseller de pencere içerisine girecektir. Herhangi bir seçim yöntemi de olmadığı için bu durum sınıflandırma sonuçları üzerinde olumsuz bir etki oluşturabilir. Zhang vd. test pikseli ile komşu piksellerin benzerliğine göre piksellere farklı ağırlıklar atayan NLW-JSRC (NonLocal Weighted-Joint Sparse Representation 
Classifier) modelini (Zhang, Li, Huang ve Zhang, 2014) geliştirmişlerdir. Bu sayede test pikseline benzer piksellerin ağırlı̆̆ 1 sınıflandırma işleminde fazla olurken, farklı karakteristiğe sahip komşu piksellerin ağırlı̆̆ 1 düşük ya da sıfir olabilmektedir. Sonuç olarak da sınıflandırma doğruluklarının JSRC modeline göre daha iyi olduğu gözlemlenmiştir.

$\mathrm{Bu}$ çalışmada, sınıflandırma aşamasında test pikseli ile birlikte hangi komşu piksellerin seçileceğine veya seçilmeyeceğine karar verebilmek için Öklit Uzaklığı, Spektral Açı Haritalama ve Pearson Korelasyon Katsayıs1 spektral eşleştirme yöntemleri ortak bir şekilde kullanılmıştır. $\mathrm{Bu}$ yöntemler birbirlerine benzer hiperspektral bantların seçimi ve sınıflandırma çalışmalarında kullanılmaktadır (Keshava, 2004; Tu vd., 2018; Yan, Cui ve Prasad, 2018). Eşleştirme metotları, test pikseli ile sırasıyla tüm komşu piksellere uygulanarak eşik değerine göre ilgili komşu pikselin seçilmesi veya seçilmemesi sağlanmıştır.

Makalenin 2. bölümünde seyrek gösterim ve ortak seyrek gösterim tabanlı sinıflandırıcılar anlatılmıştır. 3. bölümde, ortak seyrek gösterim tabanlı sinıflandırıcının performansını arttırmak için kullanılan spektral eşleştirme metotları verilmiştir. 4. bölümde önerilen metodun teorik altyapısı ve formülasyonu sunulmuştur. Deneysel kurulum ve deney sonuçları 5 bölümde verilmiştir. Son olarak bölüm 6 ise sonuç kısmıdır.

\section{YÖNTEM}

Bu bölümde sırası ile seyrek gösterim ve ortak seyrek gösterim tabanlı siniflandırıcıların teorik alt yapısı anlatılmaktadır.

\subsection{Seyrek Gösterim Tabanlı Sınıflandırıcı}

Hiperspektral görüntü içerisindeki bir piksel p, görüntünün sahip olduğu bant sayısı $B$ uzunluğundaki bir vektör olarak tanımlanır ve $\mathbf{p} \in R^{B \times 1}$ ile ifade edilir. Tüm sinıflara ait eğitim örneklerinden oluşan sözlük matrisi ise $\mathbf{D}=\left[\mathbf{D}_{1}, \mathbf{D}_{2}, \ldots, \mathbf{D}_{M}\right] \in R^{B \times N}$ ile gösterilir. Burada $M$ toplam sinif sayısinı ve $N$ toplam eğitim örneği sayısını temsil etmektedir. Seyrek gösterim tabanlı sinıflandırma modelinde bir test pikselinin sözlük matrisi içerisindeki eğitim örneklerinden yalnızca birkaçı ile temsil edilebileceği varsayıldığ 1 için bu durum

$\mathbf{p}=\mathbf{D} \boldsymbol{\alpha}+\mathbf{n}$

eşitliği ile gösterilebilir. Denklem (1)'de $\alpha=\left[\alpha_{1}, \alpha_{2}, \ldots, \alpha_{M}\right]^{T} \in R^{N \times 1} \quad$ seyrek katsay1 vektörünü ve $\mathbf{n}$ ise görüntü içerisinde olabilecek bazı gürültüleri temsil etmektedir. Seyrek gösterim tabanlı yaklaşımlarda amaç seyrek katsayı vektörünü hesaplayarak geri çatılmış test pikselini bulmaktır. Seyrek katsayı vektörünü bulmak için

$$
\hat{\boldsymbol{\alpha}}=\operatorname{argmin}\|\mathbf{p}-\mathbf{D} \boldsymbol{\alpha}\|_{2} \text { öyle } k i \quad\|\boldsymbol{\alpha}\|_{0} \leq L
$$

denklem (2)'deki optimizasyon problemi çözülmelidir. Burada $\|\cdot\|_{0}$ normu $\boldsymbol{\alpha}$ içerisindeki sıfır olmayan katsayıları sayar ve $L$ ise seyreklik seviyesinin üst sınırını yani seyrek katsayı vektörü $\hat{\alpha}$ içerisindeki sıfır olmayan katsayıları temsil eder. (2) nolu problemin çözümü için literatürdeki Dik Eşleştirme Arayış (Orthogonal Matching Pursuit - OMP) (Tropp ve Gilbert, 2007) algoritmas1 kullanılabilir.

Seyrek katsayı vektörü bulunduktan sonra test pikseli p'nin ait olduğu sınıfı bulmak için $\mathbf{p}$ ile sözlük matrisi $\mathbf{D}$ ve seyrek katsay vektörü $\hat{\alpha}$ kullanılarak artık değer

$$
E_{m}(\mathbf{p})=\left\|\mathbf{p}-\mathbf{D}_{m} \hat{\boldsymbol{\alpha}}_{m}\right\|_{2} \quad, \quad m=1,2, \cdots, M
$$

denklem (3)'teki gibi her sınıfa ait eğitim örnekleri için hesaplanır. Son olarak test pikseli p 'nin sınıfı ise artık değerin minimum olduğu eğitim örneğinin sınıfı olarak (4)'teki gibi belirlenir.

$$
\hat{m}=\operatorname{argmin}\left(E_{m}\right)
$$

\subsection{Ortak Seyrek Gösterim Tabanlı Sinıflandırıcı}

Seyrek gösterim tabanlı sinıflandırıcı (Sparse Representation based Classifier - SRC) modelinde piksellerin spektral bilgisi kullanılarak sınıflandırma işlemi yapılmaktadır. Ortak seyrek gösterim tabanlı siniflandiric1 (Joint Sparse Representation Classifier - JSRC) modelinde ise görüntü içerisindeki spektral bilgiye ek olarak uzamsal bilgi de kullanılır. Yapılan bir çalışmada test 
pikseli etrafinda sabit boyutlu bir pencere oluşturularak komşu piksellerin de aynı sınıfa ait olacağ 1 fikri ile tüm pikseller ortak bir şekilde sınıflandırılmıştır (Chen ve diğerleri, 2011). Test pikseli $\mathbf{p}_{1}{ }^{\prime}$ in $K=Q \times Q$ boyutlu kare bir penceredeki komşulukları $\mathbf{P}=\left[\mathbf{p}_{1}, \mathbf{p}_{2}, \ldots, \mathbf{p}_{Q \times Q}\right]$ ile gösterilebilir. SRC modelinde bir test pikseli için (1)'de uygulanan denklem test pikseli ile komşu piksellerin birleşiminden oluşan $\mathbf{P}$ matrisi için

$\mathbf{P}=\mathbf{D A}+\mathbf{N}$

ile ifade edilebilir. Denklem (5)'te $\mathbf{A}=\left[\alpha_{1}, \alpha_{2}, \ldots, \alpha_{Q \times Q}\right] \in R^{N \times K} \quad$ seyrek katsay 1 matrisini ve $\mathbf{N}$ ise gürültüleri temsil eder. $\mathbf{A}$ matrisini bulmak için

$$
\hat{\mathbf{A}}=\operatorname{argmin}\|\mathbf{P}-\mathbf{D A}\|_{F} \quad \text { öyle } k i \quad\|\mathbf{A}\|_{0} \leq L
$$

denklem (6)'daki problem çözülmelidir. Çözüm için Eşzamanlı Dik Eşleştirme Arayış (Simultaneous Orthogonal Matching Pursuit SOMP) (Chen ve diğerleri, 2011) algoritmas1 kullanılabilir. $\hat{\mathbf{A}}$ matrisi bulunduktan sonra $\operatorname{art} 1 \mathrm{k}$ değer

$$
E_{m}(\mathbf{P})=\left\|\mathbf{P}-\mathbf{D}_{m} \hat{\mathbf{A}}_{m}\right\|_{F} \quad, \quad m=1,2, \cdots, M
$$

(7) numaralı denklem yardımı ile her sınıfa ait eğitim örnekleri için hesaplanır. Son olarak pencere içerisindeki piksellerin sınıfi $\hat{m}$ ise minimum artık değer kullanılarak (8) ile bulunur.

$\hat{m}=\operatorname{argmin}\left(E_{m}\right)$

\subsection{Spektral Eşleştirme Metotları}

$\mathrm{Bu}$ bölümde ortak seyrek gösterim tabanlı sınıflandırıcı modelinin geliștirilmesinde kullanılan Öklit Uzaklığı, Spektral Açı Haritalama ve Pearson Korelasyon Katsayısı metotları açıklanmış ve ilgili formüller verilmiştir.

\subsection{1. Öklit uzaklığı}

$B$ boyutlu bir uzayda $X$ ve $Y$ vektörleri arasındaki uzaklığı belirlemek için Öklit Uzaklığı (Euclidean Distance - ED) kullanılmaktadır. Uzaklık değerinin küçük olması iki vektörün birbirine olan yakınlığını ifade etmektedir. Öklit uzaklığı

$$
E D(X, Y)=\sqrt{\sum_{i=1}^{B}\left(X_{i}-Y_{i}\right)^{2}}
$$

(9)'daki formül yardımı ile hesaplanabilir.

\subsubsection{Spektral açı haritalama}

Spektral Açı Haritalama (Spectral Angle Mapper - SAM) yöntemi sayesinde $B$ boyutlu bir spektral uzayda $X$ ve $Y$ vektörleri arasındaki açı hesaplanabilir. Açı değerinin küçük olması iki vektörün benzer olduğunu göstermektedir. $S A M$ değeri takip eden denklem yardımı ile hesaplanabilir.

$$
\operatorname{SAM}(X, Y)=\cos ^{-1}\left(\frac{\sum_{i=1}^{B} X_{i} Y_{i}}{\sqrt{\sum_{i=1}^{B} X_{i}^{2}} \sqrt{\sum_{i=1}^{B} Y_{i}^{2}}}\right)
$$

\subsubsection{Pearson korelasyon katsayısı}

Pearson Korelasyon Katsayis1 (Pearson Correlation Coefficient - PCC) da SAM yöntemi gibi iki vektörün benzerliğini gösterir ve -1 ile 1 arasında bir değerdir. Değerin -1'e yakın olması iki vektörün benzer olduğunu fakat zit yönde olduğunu belirtir. Tam tersi durumda yani değerin 1'e yakın olması ise iki vektörün benzer olduğunu ve aynı yönde olduğunu belirtir. Bu sebeple eğer $B$ boyutlu bir uzayda $X$ ve $Y$ vektörleri birbirlerine benzer ise mutlak değer 1'e yakın olmaktadır. Tam tersi durumda ise bu değer 0'a yakın olmaktadır.

$$
P C C(X, Y)=\frac{\sum_{i=1}^{B}\left(X_{i}-\bar{X}\right)\left(Y_{i}-\bar{Y}\right)}{\sqrt{\sum_{i=1}^{B}\left(X_{i}-\bar{X}\right)^{2}} \sqrt{\sum_{i=1}^{B}\left(Y_{i}-\bar{Y}\right)^{2}}}
$$

Denklem (11)'de $\quad \bar{X}=\left(\frac{1}{-} \sum_{i=1}^{B} X_{i}\right) \quad$ ve $\bar{Y}=\left(\frac{1}{{ }_{B}} \sum_{i=1}^{B} Y_{i}\right)$ sirasıyla $X$ ve $Y$ vektörlerinin ortalama değerlerini temsil etmektedir.

\subsection{Problem Formülasyonu}

Pencere içerisindeki pikseller $\mathbf{p}_{1}$ test pikseli olmak koşulu ile $\mathbf{P}=\left[\mathbf{p}_{1}, \mathbf{p}_{2}, \ldots, \mathbf{p}_{Q \times Q}\right]$ matrisi 


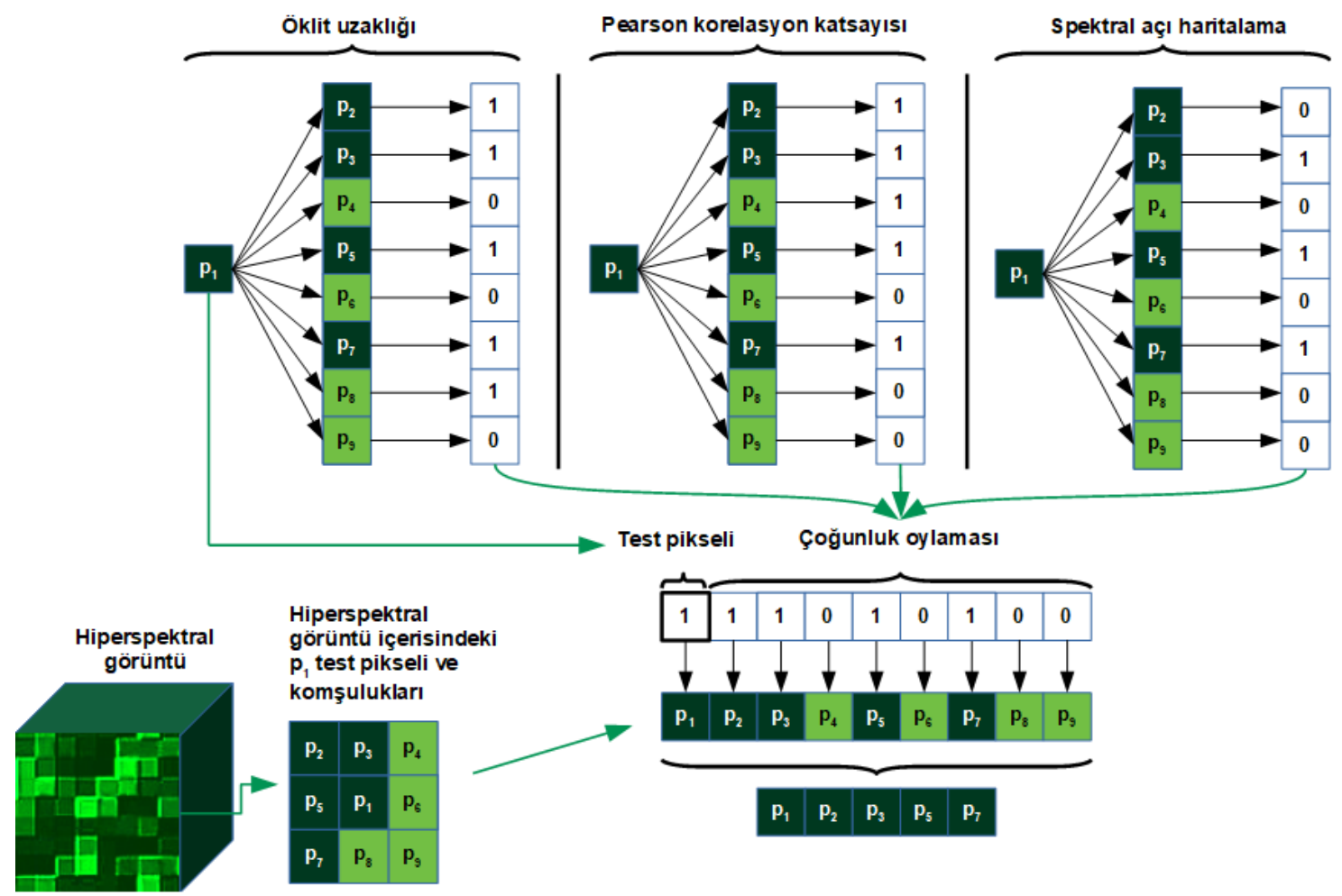

Şekil 1. Test Pikseli Etrafında $3 \times 3$ Boyutunda Oluşturulan Pencere İçerisindeki Komşu Piksellerin Öklit Uzaklığı, Pearson Korelasyon Katsayısı ve Spektral Açı Haritalama Yöntemleri ile Seçilmesi

ile gösterilir. $\mathbf{p}_{1}$ pikseli sırası ile pencere içerisindeki tüm komșu pikseller ile $E D, S A M$ ve $P C C$ yöntemlerine tabi tutulur. Bu durumu

$$
\begin{aligned}
& E D_{j}=E D\left(\mathbf{p}_{1}, \mathbf{p}_{j}\right) \quad j=2,3, \cdots, Q \times Q \\
& S A M_{j}=\operatorname{SAM}\left(\mathbf{p}_{1}, \mathbf{p}_{j}\right) \quad j=2,3, \cdots, Q \times Q \\
& P C C_{j}=P C C\left(\mathbf{p}_{1}, \mathbf{p}_{j}\right) \quad j=2,3, \cdots, Q \times Q
\end{aligned}
$$

Denklem (12), (13) ve (14)'teki gibi gösterebiliriz. Bu değerler tüm pikseller için bulunduktan sonra değerlerin ortalaması (15), (16) ve (17) numaralı denklemler aracılığı ile hesaplanir.

$\overline{E D}=\frac{1}{(Q \times Q)-1} \sum_{j=2}^{Q \times Q} E D_{j}$

$\overline{S A M}=\frac{1}{(Q \times Q)-1} \sum_{j=2}^{Q \times Q} S A M_{j}$

$\overline{P C C}=\frac{1}{(Q \times Q)-1} \sum_{j=2}^{Q \times Q} P C C_{j}$

Ortalama değerler de bulunduktan sonra test pikseli ve ilgili komşu piksel için hesaplanan spektral eşleştirme metotlarının eşik değeri olarak belirlenen ortalama değerlerden büyük veya küçük olması durumuna göre yalnızca $[0,1]$ değerlerini alan

$$
\begin{aligned}
& \mathbf{E D}_{1,0}= \begin{cases}1 \quad ; \quad E D_{j} \geq \overline{E D} \\
0 ; & E D_{j}<\overline{E D}\end{cases} \\
& \mathbf{S A M}_{1,0}= \begin{cases}1 \quad & S A M_{j} \geq \overline{S A M} \\
0 & ; \quad S A M_{j}<\overline{S A M}\end{cases}
\end{aligned}
$$

$\mathbf{P C C}_{1,0}= \begin{cases}1 \quad ; \quad P C C_{j} \geq \overline{P C C} \\ 0 \quad ; \quad P C C_{j}<\overline{P C C}\end{cases}$

$\mathbf{E D}_{1,0}, \quad \mathbf{S A M}_{1,0} \quad$ ve $\quad \mathbf{P C C}_{1,0}$ vektörleri oluşturulur. Daha sonra bu üç vektör üzerine çoğunluk oylaması (Majority Voting - $M V$ ) kuralı uygulanarak 1 ve 0 'lardan oluşan $\mathbf{V}$ vektörü oluşturulur.

$$
\begin{aligned}
\mathbf{V} & =M V\left[\mathbf{E D}_{1,0} ; \mathbf{S A M}_{1,0} ; \mathbf{P C C}_{1,0}\right] \\
& =\left[V_{2} V_{3} \cdots V_{Q \times Q}\right] \quad ; \quad V_{j=2,3, \cdots, Q \times Q} \in[0,1]
\end{aligned}
$$


Denklem (21)'de merkez test pikseli $\mathbf{p}_{1}$ etrafindaki komşu pikseller için seçim yapılmaktadır. $\mathbf{p}_{1}$ pikselini de hesaba katarak ağırlık vektörü $\mathbf{W}$

$\mathbf{W}=\left[1 V_{2} V_{3} \cdots V_{Q \times Q}\right]$

oluşturulur. Son olarak ise test pikseli $\mathbf{p}_{1}$ ve seçilen komşu pikselleri içeren $\tilde{\mathbf{P}}$ matrisi

$$
\tilde{\mathbf{P}}=\mathbf{W P}
$$

(23) nolu denklem yardımı ile oluşturulur. $\mathrm{Bu}$ işlemden sonra sırasıyla (5), (6), (7) ve (8) numaralı denklemler hesaplanarak pencere içerisindeki piksellerin sınıfi belirlenir.

Merkez test pikseli etrafındaki komşu piksellerin önerilen spektral eşleştirme metotları yardımı ile seçilmesini gösteren görsel Şekil 1'de bulunmaktadır. Ayrıca önerilen metoda ait aşamalar Algoritma 1'de özetlenmiştir.

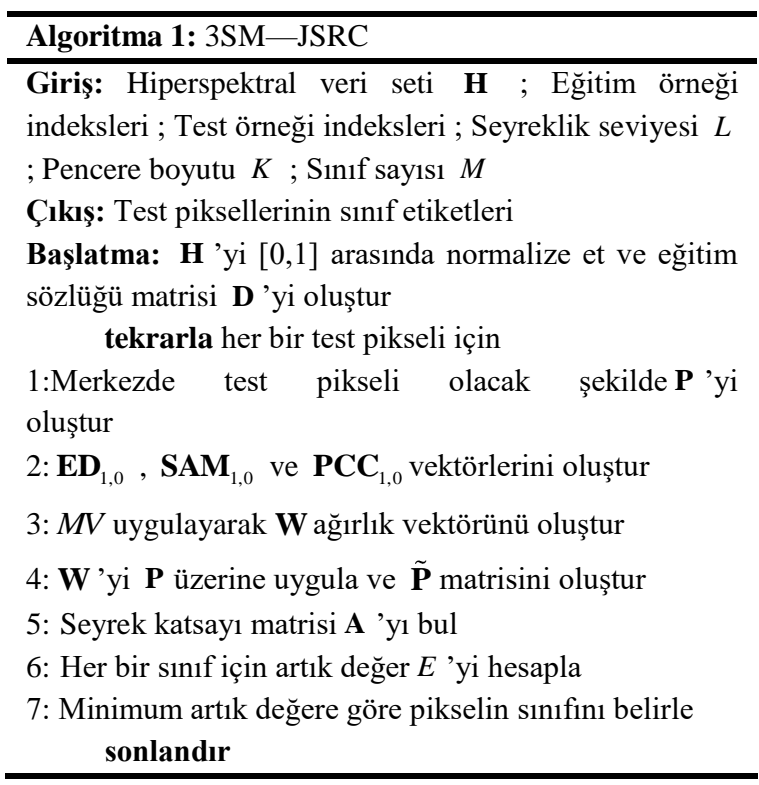

\section{BULGULAR}

Bu bölümde, önerilen 3SM-JSRC metodu iki adet hiperspektral veri seti üzerinde test edilmiștir. Literatürdeki SVM (Melgani ve Bruzzone, 2004), SRC (Chen vd., 2011), JSRC (Chen vd., 2011) ve NLW-JSRC (Zhang vd., 2014) metotları ise karşılaştırma amacı ile kullanılmıştır. $\mathrm{Bu}$ çalışma kapsamında yapılan tüm deneyler farklı eğitim örnekleri ile $10 \mathrm{kez}$ tekrarlanmıştır ve sonuçların ortalaması alınmıştır. Elde edilen sınıflandırma sonuçlarını karşılaştırmak için

Tablo 1. Indian Pines Veri Setinde Her Sinıfa Ait Eğitim ve Test Örnekleri Sayısı

\begin{tabular}{|c|c|c|c|}
\hline Sinıf & İsim & Eğitim & Test \\
\hline 1 & Alfalfa & 6 & 48 \\
\hline 2 & Corn-notill & 144 & 1290 \\
\hline 3 & Corn-min & 84 & 750 \\
\hline 4 & Corn & 24 & 210 \\
\hline 5 & Grass/Pasture & 50 & 447 \\
\hline 6 & Grass/Trees & 75 & 672 \\
\hline 7 & Grass/Pasture-mowed & 3 & 23 \\
\hline 8 & Hay-windrowed & 49 & 440 \\
\hline 9 & Oats & 2 & 18 \\
\hline 10 & Soybean-notill & 97 & 871 \\
\hline 11 & Soybean-min & 247 & 2221 \\
\hline 12 & Soybean-clean & 62 & 552 \\
\hline 13 & Wheats & 22 & 190 \\
\hline 14 & Woods & 130 & 1164 \\
\hline 15 & Building-Grass-Tree-Drives & 38 & 342 \\
\hline 16 & Stone-steel Towers & 10 & 85 \\
\hline & Toplam & 1043 & 9323 \\
\hline
\end{tabular}

ise bazı hesaplama metrikleri

kullanılmaktadır. Bunlar sirasiyla genel doğruluk $(G D)$, sınıf doğrulukları $(S D)$, ortalama doğruluk $(O D)$, ve kappa $(\mathrm{K})$ değerleridir. $\mathrm{Bu}$ metrikler ise aşağıdaki gibi hesaplanır.

$G D=\frac{\text { Doğru sinıflandırılan test örneği sayısı }}{\text { Toplam test örneği sayısı }}$

$S D=\frac{\text { Sinıfa doğru atanan test örneği sayıs1 }}{\text { Sınıftaki toplam test örneği sayısı }}$

$O D=\frac{\text { Sınıf doğruluk değerlerinin toplamı }}{\text { Toplam sınıf sayısı }}$

$\mathrm{K}=\frac{G D-\text { Beklenen doğruluk değeri }}{1-\text { Beklenen doğruluk değeri }}$

\subsection{Indian Pines}

$\mathrm{Bu}$ veri seti Indian Pines test alanı üzerinde AVIRIS (Airborne/Visible Infrared Imaging Spectrometer) sensörü tarafindan elde edilmiştir. Toplamda 224 adet spektral banttan oluşmaktadır ve bantların dalgaboyu 0.4-2.5 $\mu \mathrm{m}$ arasındadır. Su emilimi gibi sebeplerden dolay1 24 adet bant (104-108, 150-163, 220) veri setinden çıkartılmıştır (Gualtieri ve Cromp, 1999) ve dolayısıyla geriye 200 adet spektral bant kalmıştır. 
Uzamsal olarak ise $145 \times 145$ adet pikselden oluşmaktadır. Çoğunluğu tarım ürünlerinden

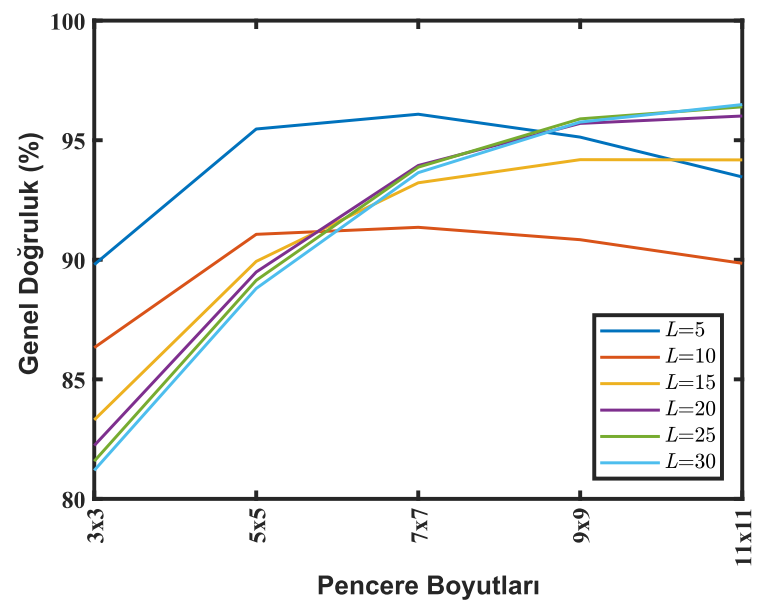

(a) oluşan 16 adet sınıf içermektedir. Bu veri seti ile ilgili yapılan tüm çalışmalarda her sınıftan

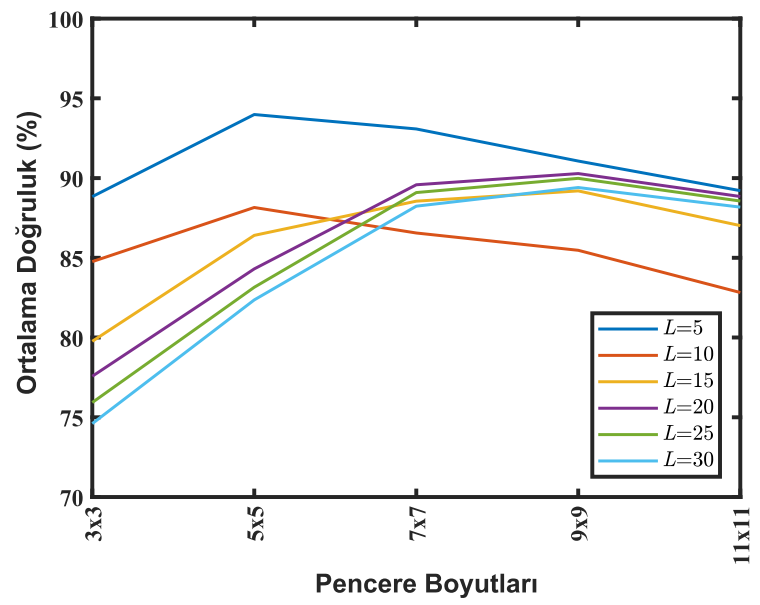

(b)

Şekil 2. Indian Pines Veri Setinde Farklı Pencere Boyutları ve Seyreklik Seviyeleri Kullanılarak Elde Edilen (a) $G D$, (b) $O D$ Sonuçları

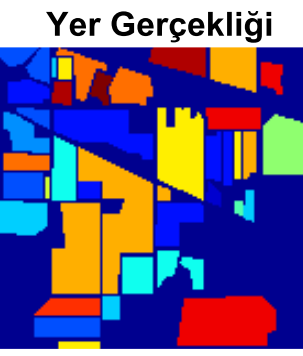

(a)

$\% 95.93$

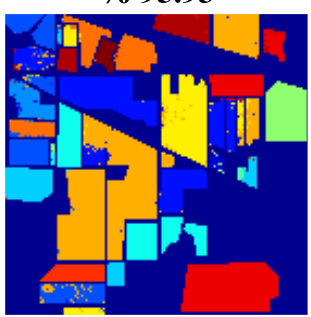

(e)
$\% 84.57$

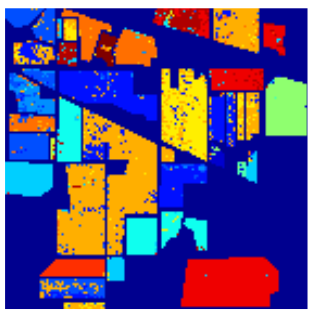

(b)

$\% 96.33$

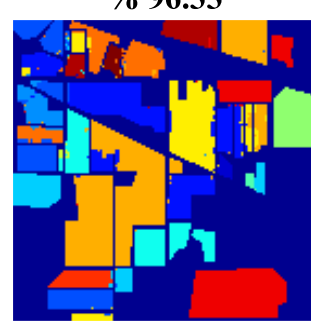

(f)
$\% 75.63$

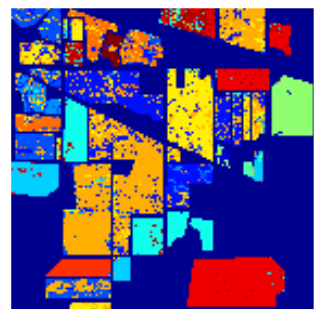

(c)
$\% 95.21$

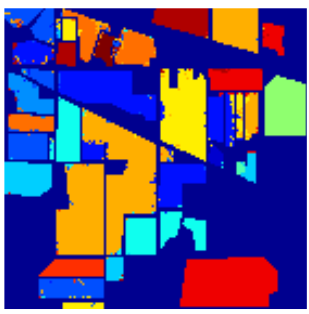

(d)

Şekil 3. Indian Pines Görüntüsüne Ait: (a) Yer Gerçekliği Haritas1; (b) SVM, (c) SRC, (d) JSRC, (e) NLW-JSRC, (f) 3SM-JSRC Metotları ile Elde Edilen Sınıflandırma Haritaları ve GD Sonuçları; (g) Sinıf İsimleri

\%10 kadar örnek eğitim için ve geriye kalan \%90 örnek ise test için kullanılmıştır. Sınıflara ve örneklere ait bilgiler Tablo 1'de bulunmaktadır.

SVM için Gauss RBF çekirdeği kullanılmış ve en iyi parametrelerin seçilebilmesi için 5-kat çapraz doğrulama yapılmıştır. SRC metodunda (Chen vd., 2011)'te önerilen $L=5$ seyreklik seviyesi kullanılmıştır. JSRC metodu için $9 \times 9$ pencere boyutu ve seyreklik seviyesi olarak 30 kullanılmıştır (Chen vd., 2011).NLW-JSRC metodunda da en yüksek genel doğruluk değerlerine komşuluk için pencere boyutu $9 \times 9$, yerel olmayan ağırlık hesaplaması için kullanılan pencere boyutu $7 \times 7$ ve seyreklik seviyesi olarak 30 değerleri ile ulaş1lmıştır (Zhang vd., 2014). 
Önerilen 3SM-JSRC metodu için optimum seyreklik seviyesi ve pencere boyutunun belirlenebilmesi için farklı değerler ile test yapılmıştır. Pencere boyutu olarak $3 \times 3,5 \times 5$, $7 \times 7,9 \times 9$ ve $11 \times 11$ kullanılmıştır. Seyreklik seviyesi olarak da $5,10,15,20,25$ ve 30 değerleri kullanılmıştır.

Şekil 2'de ise bu parametreler ile elde edilen $G D$ ve $O D$ sonuçları bulunmaktadır. Görüleceği üzere $9 \times 9$ pencere boyutu ile $11 \times 11$ pencere boyutu arasında tüm seyreklik seviyeleri için $O D$ sonuçlarında düşüş olmaktadir. $3 \times 3$ pencere boyutu ile $5 \times 5$ pencere boyutu arasinda ise hem $G D$ hem de $O D$ sonuçlarında artış gözlemlenmektedir. Dolayısıyla pencere boyutu için $5 \times 5,7 \times 7$ veya $9 \times 9$ arasında seçim yapılmalıdır. $9 \times 9$ ölçekte 25 seyreklik seviyesinde $G D$ sonucu $5 \times 5$ ve $7 \times 7$ ölçeklerin sonuçlarına yakın çıksa da $O D$ sonucu diğer iki ölçek sonuçlarına göre düşük çıkmaktadır.

Tablo 2. Indian Pines Veri Seti için SVM, SRC, JSRC, NLW-JSRC ve 3SM-JSRC Metotları Kullanılarak Elde Edilen Karşılaştırma Sonuçları (\%)

\begin{tabular}{cccccc}
\hline Sinıf & SVM & SRC & JSRC & NLW-JSRC & 3SM-JSRC \\
\hline 1 & 76.67 & 68.33 & 86.67 & 85.83 & $\mathbf{9 6 . 8 8}$ \\
2 & 81.01 & 65.15 & 93.74 & $\mathbf{9 4 . 5 3}$ & 93.90 \\
3 & 76.65 & 61.55 & 89.91 & 89.29 & $\mathbf{9 3 . 6 8}$ \\
4 & 71.00 & 43.33 & $\mathbf{9 3 . 8 1}$ & 91.52 & 89.67 \\
5 & 94.97 & 89.08 & 93.42 & $\mathbf{9 7 . 2 3}$ & 96.00 \\
6 & 95.67 & 94.94 & 99.14 & $\mathbf{9 9 . 8 8}$ & 98.44 \\
7 & 79.57 & 82.17 & 51.74 & 33.04 & $\mathbf{8 6 . 9 6}$ \\
8 & 97.48 & 97.25 & 99.80 & $\mathbf{1 0 0}$ & $\mathbf{1 0 0}$ \\
9 & $\mathbf{7 1 . 1 1}$ & 41.67 & 4.44 & 23.89 & 51.67 \\
10 & 70.54 & 72.45 & 89.07 & 86.99 & $\mathbf{9 2 . 9 2}$ \\
11 & 85.41 & 76.47 & 97.25 & $\mathbf{9 8 . 0 4}$ & 97.35 \\
12 & 86.68 & 59.22 & 89.00 & $\mathbf{9 4 . 4 9}$ & 91.47 \\
13 & 99.16 & 97.74 & 99.21 & $\mathbf{9 9 . 7 4}$ & 98.79 \\
14 & 95.40 & 93.77 & 99.24 & 99.24 & $\mathbf{9 9 . 6 6}$ \\
15 & 61.87 & 44.71 & $\mathbf{9 8 . 4 5}$ & 93.71 & 94.74 \\
16 & 89.06 & 87.53 & 92.24 & $\mathbf{9 8 . 2 4}$ & 90.94 \\
\hline$G D$ & 84.83 & 75.91 & 94.86 & 95.31 & $\mathbf{9 5 . 8 3}$ \\
$O D$ & 83.26 & 73.46 & 86.07 & 86.60 & $\mathbf{9 2 . 0 6}$ \\
K & 82.69 & 72.49 & 94.13 & 94.64 & $\mathbf{9 5 . 2 4}$ \\
\hline
\end{tabular}

Dolayısıyla seçim için yalnızca $5 \times 5$ ve $7 \times 7$ ölçek boyutları kalmaktadır. Her iki ölçek boyutu için de $L=5$ seyreklik seviyesinde $G D$ ve $O D$ sonuçları daha yüksek çıkmaktadır. $7 \times 7$ ölçek boyutunda $G D$ sonucu daha yüksek olduğu için optimum parametreler $7 \times 7$ pencere boyutu ve $L=5$ seyreklik seviyesi olarak belirlenmiştir. Çünkü yüksek $G D$ sonucu doğru sınıflandırılan test örneklerinin sayısının daha fazla olduğunu işaret etmektedir.

Parametre ayarlamaları ve deneyler yapıldıktan sonra elde edilen karşılaştırma sonuçları Tablo 2'de sunulmuştur. Görüleceği üzere önerilen 3SM-JSRC metodu $G D, O D$ ve $\mathrm{K}$ değerleri açısından diğer metotlara göre üstün gelmektedir. Herhangi bir şekilde komşuluk bilgilerinin kullanılmadığ $\mathrm{SVM}$ ve SRC yöntemlerinde sınıflandırma doğruluk sonuçları komşuluk bilgilerinin kullanıldığı JSRC, NLW-JSRC ve 3SM-JSRC yöntemlerine göre daha düşük olmaktadır. Dolayısıyla komşuluk bilgileri sayesinde sınıflandırma doğruluk sonuçlarının arttığ rahatlıkla gözlemlenebilir. Pencere içerisindeki komşu pikseller için herhangi bir seçimin yapılmadığı JSRC metodu ile komşu pikseller için seçimin yapıldı ğ 13 SM-JSRC metodunun sonuçları karşılaştırılacak olursa $G D$ açısından yaklaşık olarak \%1, $O D$ açısından yaklaşık olarak \%6 ve $\mathrm{K}$ açısından \%1'den daha yüksek sınıflandırma doğruluk sonuçları 3SMJSRC ile elde edilmektedir. Komşu piksellere ağırlık atamasının yapıldığı NLW-JSRC metoduna göre de 3SM-JSRC metodunda $G D$ ve $\mathrm{K}$ değerlerinde ciddi bir farklılık olmasa da $O D$ açısından metodumuz \%6'ya yakın daha üstün gelmektedir.

Sınıflandırma sonuçlarının görsel olarak daha iyi bir şekilde gözlemlenebilmesi için Indian Pines verisine ait yer gerçekliği haritası, karşılaştırma yapılan metotlar ve önerilen metoda ait sınıflandırma haritaları Şekil 3'de sunulmuştur. Görüleceği üzere 3SM-JSRC metoduna ait sınıflandirma haritası benzerlik açısından yer gerçekliği haritası ile daha çok örtüşmektedir.

\subsection{University of Pavia}

$\mathrm{Bu}$ veri seti ROSIS (Reflective Optics System Imaging Spectrometer) sensörü tarafindan Pavia Üniversitesi üzerinde yapılan uçuş ile elde edilmiştir. Toplamda 103 adet spektral banttan oluşmaktadır. Orijinal görüntü $610 \times 610$ adet piksele sahip olsa da veri seti içerisindeki bazı kısımlar hakkında herhangi bir bilgi olmadığı için veri seti içerisinden çıkartılmıştır ve sınıflandırma amacı ile yapılan çalışmalarda $610 \times 340$ uzamsal boyut 
kullanılmıştır. Farklı farklı gruplara ait (asfalt, çimenler, ağaçlar, toprak vs.) 9 adet siniftan oluşmaktadır. Indian Pines veri setinde olduğu

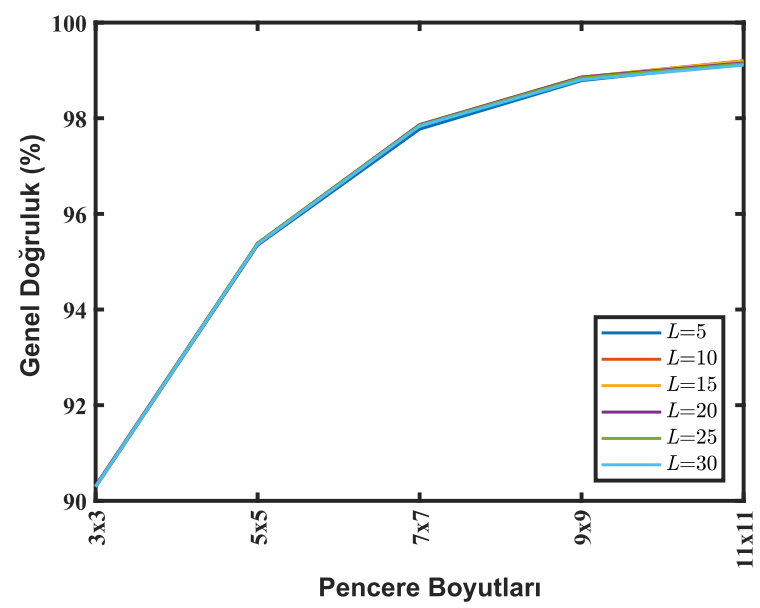

(a) gibi bu veri seti ile ilgili yapılan tüm çalışmalarda da her sınıftan \%10 kadar örnek

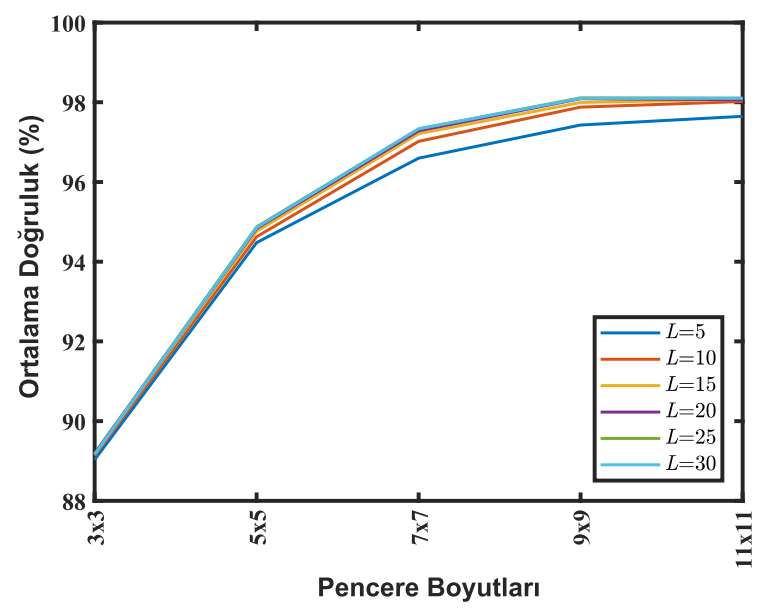

(b)

Şekil 4. University of Pavia Veri Setinde Farklı Pencere Boyutları ve Seyreklik Seviyeleri Kullanılarak Elde Edilen (a) GD, (b) OD Sonuçları

eğitim için ve geriye kalan \%90 örnek ise test için kullanılmıştır. Sınıflara ve örneklere ait bilgiler Tablo 3'de bulunmaktadır.

Tablo 3. University of Pavia Veri Setinde Her Sınıfa Ait Eğitim ve Test Örnekleri Sayısı

\begin{tabular}{cccc}
\hline Sinıf & İsim & Eğitim & Test \\
\hline 1 & Asphalt & 664 & 5967 \\
2 & Meadows & 1865 & 16784 \\
3 & Gravel & 210 & 1889 \\
4 & Trees & 307 & 2757 \\
5 & Painted metal sheets & 135 & 1210 \\
6 & Bare Soil & 503 & 4526 \\
7 & Bitumen & 133 & 1197 \\
8 & Self-Blocking Bricks & 369 & 3313 \\
9 & Shadows & 95 & 852 \\
\hline \multicolumn{4}{c}{}
\end{tabular}

SVM için Gauss RBF çekirdeği kullanılmış ve en iyi parametrelerin seçilebilmesi için 5-kat çapraz doğrulama yapılmıştır. SRC metodunda $L=5$ seyreklik seviyesi kullanılmıştır. JSRC metodu için $3 \times 3$ pencere boyutu ve seyreklik seviyesi olarak 10 kullanılmıştır (Zhang vd., 2014). NLW-JSRC metodunda da en yüksek genel doğruluk değerlerine komşuluk için pencere boyutu $5 \times 5$, yerel olmayan ağırlık hesaplaması için kullanılan pencere boyutu $7 \times 7$ ve seyreklik seviyesi olarak 15 değerleri ile ulaşılmıştır (Zhang vd., 2014). Önerilen
3SM-JSRC metodu için optimum seyreklik seviyesi ve pencere boyutunun belirlenebilmesi için farklı değerler ile test yapılmıştır. Pencere boyutu olarak $3 \times 3,5 \times 5$, $7 \times 7,9 \times 9$ ve $11 \times 11$ kullanılmıştır. Seyreklik seviyesi olarak da $5,10,15,20,25$ ve 30 değerleri kullanılmıştır.

Şekil 4'de ise bu parametreler ile elde edilen $G D$ ve $O D$ sonuçları bulunmaktadır. Görüleceği üzere tüm seyreklik seviyeleri için de pencere boyutu arttıkça hem $G D$ hem de $O D$ sonuçları artmaktadır. Fakat $9 \times 9$ ölçekten $11 \times 11$ ölçeğe doğru geçiş yaparken $O D$ sonuçlarındaki artış iyice azalmıştır. Şekil 4(a) dikkatli incelenecek olursa $11 \times 11$ ölçekte $L=15$ seyreklik seviyesi için $G D$ sonucu en yüksek değere ulaşmaktadır. $O D$ sonuçlarında ise $11 \times 11$ ölçekte $L=5$ seyreklik seviyesi haricindeki değerler neredeyse ayn çıkmaktadır. $\mathrm{Bu}$ sebeple $11 \times 11$ pencere boyutu ve $L=15$ seyreklik seviyesi bu veri seti için optimum değerler olarak belirlenmiştir.

Parametre ayarlamalarından sonra yapılan karşılaştırma sonuçlarına ait veriler Tablo 4'de sunulmuştur. Görüleceği üzere $G D, O D$ ve $\mathrm{K}$ değerleri 3SM-JSRC metodunda diğer metotlara göre oldukça yüksek çıkmaktadır. 
JSRC ile $G D$ açısından arasındaki fark yaklaşık olarak \%7, $O D$ açısından yaklaşık olarak \%7 ve $\mathrm{K}$ açısından yaklaşık olarak $\% 9.50$ olmaktadır. NLW-JSRC ile ise $G D$ açısından aradaki fark yaklaşık olarak \%6, $O D$ açısından yaklaşık olarak $\% 6$ ve $\mathrm{K}$ açısından yaklaşık olarak \%8 olmaktadır. Komşuluk bilgilerinin kullanılmadığ SVM ve SRC metotlarında ise sinıflandırma doğruluk sonuçları diğer 3 metoda göre düşük

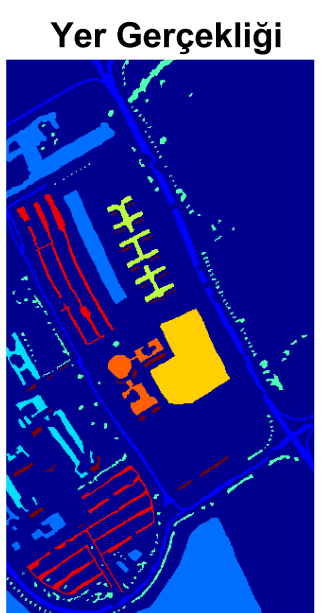

(a)

$\% 92.95$

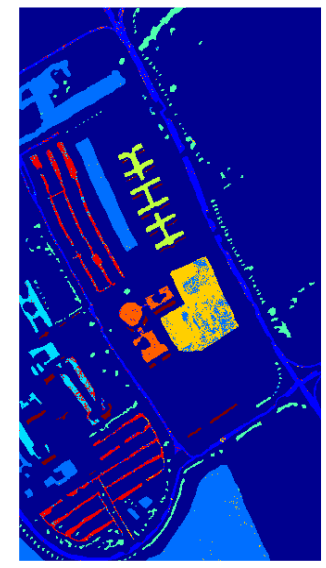

(e)

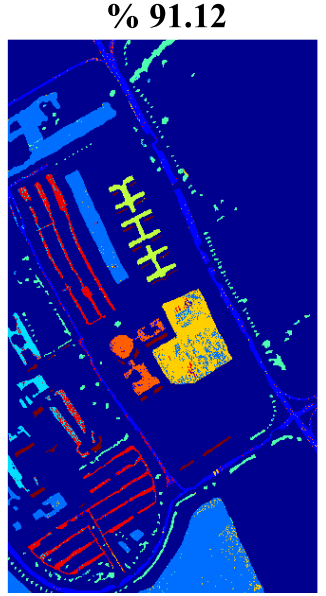

(b)

$\% 98.89$

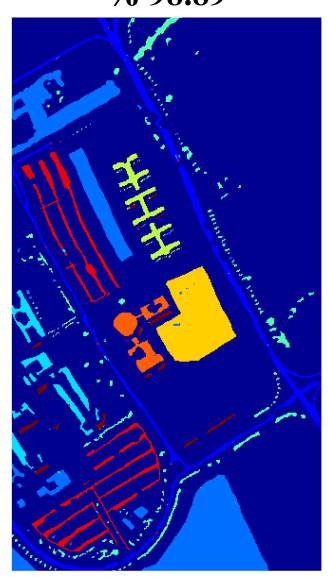

(f)

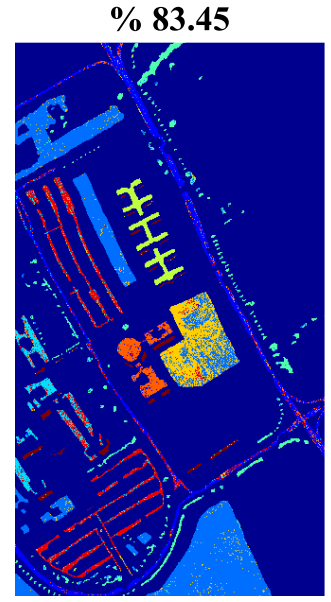

(c)

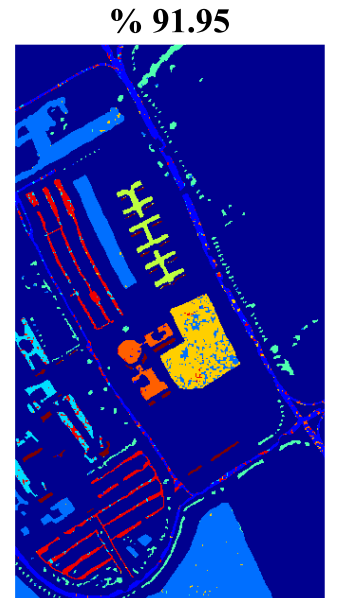

(d)

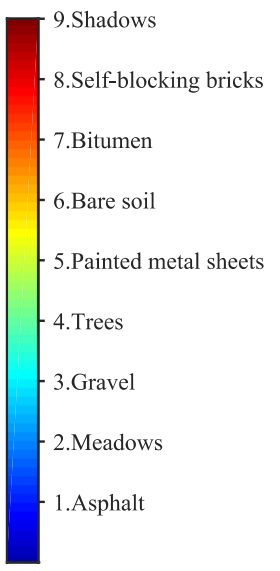

$(\mathrm{g})$

Şekil 5. University of Pavia Görüntüsüne Ait: (a) Yer Gerçekliği Haritası; (b) SVM, (c) SRC, (d) JSRC, (e) NLW-JSRC, (f) 3SM-JSRC Metotları ile Elde Edilen Sinıflandırma Haritaları ve GD Sonuçları; (g) Sınıf İsimleri

kalmaktadır. Bu veri setinde JSRC ve NLWJSRC metotlarına göre 3SM-JSRC metodunda sınıflandırma sonuçlarının daha yüksek olmasının sebebi büyük pencere boyutunun kullanılmasıdır. Çünkü JSRC ve NLW-JSRC metotlarında küçük pencere boyutlarında optimum değerlere ulaşılmıştır. Fakat küçük pencere ölçeklerinde uzamsal bilgi yeterince kullanılamayacağı için yüksek sınıflandırma doğruluk değerleri elde edilememiştir. 3SMJSRC metodunda ise yüksek pencere ölçeği sayesinde uzamsal bilgi oldukça verimli bir şekilde kullanılmıştır. Ayrıca pencere içerisine giren farklı sinıflara ait pikseller de spektral eşleştirme metotları sayesinde elenmiştir. $\mathrm{Bu}$ sayede de sınıflandırma doğruluk sonuçları oldukça yüksek değerlere ulaşmıştır.

Tablo 4. University of Pavia Veri Seti İçin SVM, SRC, JSRC, NLW-JSRC ve 3SMJSRC Metotları Kullanılarak Elde Edilen Karşılaştırma Sonuçları (\%)

\begin{tabular}{cccccc}
\hline Sinif & SVM & SRC & JSRC & NLW-JSRC & 3SM-JSRC \\
\hline 1 & 91.53 & 77.18 & 82.14 & 93.30 & $\mathbf{9 8 . 7 3}$
\end{tabular}




\begin{tabular}{cccccc}
2 & 97.19 & 94.97 & 98.79 & 99.60 & $\mathbf{9 9 . 9 4}$ \\
3 & 70.70 & 61.90 & 83.34 & 84.66 & $\mathbf{9 9 . 3 3}$ \\
4 & 93.80 & 84.54 & 91.58 & 94.60 & $\mathbf{9 5 . 5 0}$ \\
5 & 99.51 & 99.49 & $\mathbf{1 0 0}$ & $\mathbf{1 0 0}$ & 99.98 \\
6 & 75.92 & 56.36 & 79.54 & 73.29 & $\mathbf{9 9 . 5 1}$ \\
7 & 83.13 & 78.59 & 94.06 & 92.44 & $\mathbf{9 9 . 5 6}$ \\
8 & 87.60 & 76.88 & 91.34 & 83.39 & $\mathbf{9 9 . 6 2}$ \\
9 & $\mathbf{9 9 . 0 7}$ & 94.92 & 94.99 & 97.51 & 82.58 \\
\hline$G D$ & 91.12 & 83.38 & 91.84 & 92.79 & $\mathbf{9 8 . 9 3}$ \\
$O D$ & 88.72 & 80.54 & 90.64 & 90.98 & $\mathbf{9 7 . 1 9}$ \\
$\mathrm{K}$ & 88.13 & 77.64 & 89.09 & 90.30 & $\mathbf{9 8 . 5 8}$ \\
\hline
\end{tabular}

Sınıflandırma sonuçlarının görsel olarak daha iyi bir şekilde gözlemlenebilmesi için University of Pavia verisine ait yer gerçekliği haritası, karşılaştırma yapılan metotlar ve önerilen metoda ait sinıflandırma haritaları Şekil 5'te sunulmuştur. Görüleceği üzere 3SM-JSRC metoduna ait sinıflandırma haritas1 benzerlik açısından yer gerçekliği haritası ile daha çok örtüşmektedir. 3SM-JSRC metodu haricindeki metotlarda "Bare soil" sınıfına ait sonuçlar oldukça gürültülü olmasına rağmen 3SM-JSRC metodunda bu sınıfa ait sonuçlar yer gerçekliği haritası ile iyi bir şekilde örtüşmektedir. Yine aynı şekilde "Gravel" ve "Self-blocking bricks" sınıflarına ait sonuçlar sinıflandırma haritaları üzerinde incelenecek olursa 3SM-JSRC haricindeki yöntemlerde gürültü bulunmaktadır. 3SM-JSRC'de ise bu sınıfa ait sonuçlar yer gerçekliği haritasındaki ile neredeyse birebir örtüşmektedir.

\section{SONUÇLAR ve TARTIŞMA}

$\mathrm{Bu}$ çalışmada JSRC sınıflandırma modelinin başarım oranlarını arttırmak için spektral eşleştirme yöntemlerini ortaklaşa kullanan 3SM-JSRC modeli önerilmiştir. Test örneğinin sınıflandırılması aşamasında test pikseli etrafındaki komşu piksellerin spektral eşleştirme yöntemleri yardımı ile sınıflandırma işlemine dahil edilmesi veya edilmemesi sağlanmıştır. Oluşturulan sınıflandırma problemi ise SOMP yardımı ile çözülmüştür. 3SM-JSRC metodunun temel avantaj1 ise büyük pencere boyutları kullanılsa bile komşu piksellerin seçimi sayesinde farklı sınıflara ait piksellerin çoğunlukla pencere içerisinden çıkartılmasıdır. Önerilen sınıflandırma modeli iki adet hiperspektral veri seti üzerinde de test edilmiştir. Yapılan deneyler sonucunda 3SMJSRC metodunun literatürdeki benzer metotlara göre daha iyi sınıflandırma doğruluk sonuçları sağladığı gözlemlenmiştir.
Piksellerin benzerlik durumlarını daha iyi bir şekilde ortaya çıkarabilecek eşleştirme yöntemlerinin kullanılması veya farklı bir ağırlık atama metodunun geliştirilmesi ve kullanılması ile önerilen metot geliştirilebilir.

\section{KAYNAKÇA}

Bruzzone, L., Chi, M. ve Marconcini, M. (2006). A Novel Transductive SVM for Semisupervised Classification of RemoteSensing Images. IEEE Transactions on Geoscience and Remote Sensing, 44(11), 3363-3373.

Chen, Y., Nasrabadi, N. M. ve Tran, T. D. (2011). Hyperspectral Image Classification Using Dictionary-Based Sparse Representation. IEEE Transactions on Geoscience and Remote Sensing, 49(10), 3973-3985.

Chi, M. ve Bruzzone, L. (2007). Semisupervised Classification of Hyperspectral Images by SVMs Optimized in the Primal. IEEE, 45(6), 1870-1880.

Christophe, E., Leger, D. ve Mailhes, C. (2005). Quality criteria benchmark for hyperspectral imagery. IEEE, 43(9), 21032114.

Dalponte, M., Ørka, H. O., Gobakken, T., Gianelle, D. ve Næsset, E. (2013). Tree Species Classification in Boreal Forests With Hyperspectral Data. IEEE, 51(5), 26322645.

Datt, B., McVicar, T. R., Niel, T. G. V., Jupp, D. L. B. ve Pearlman, J. S. (2003). Preprocessing EO-1 Hyperion hyperspectral data to support the application of agricultural indexes. IEEE, 41(6), 1246-1259.

Dundar, T. ve Ince, T. (2019). Sparse Representation-Based Hyperspectral Image Classification Using Multiscale Superpixels and Guided Filter. IEEE Geoscience and Remote Sensing Letters, 16(2), 246-250.

Fang, L., Li, S., Kang, X. ve Benediktsson, J. A. (2014). Spectral-Spatial Hyperspectral Image Classification via Multiscale Adaptive Sparse Representation. IEEE Transactions on Geoscience and Remote Sensing, 52(12), 7738-7749.

Fang, L., Li, S., Kang, X. ve Benediktsson, J. A. (2015). Spectral-Spatial Classification of Hyperspectral Images With a SuperpixelBased Discriminative Sparse Model. IEEE Transactions on Geoscience and Remote Sensing, 53(8), 4186-4201.

Garcia, M. ve Ustin, S. L. (2001). Detection of interannual vegetation responses to climatic variability using AVIRIS data in a coastal 
savanna in California. IEEE, 39(7), 14801490.

Goel, P. K., Prasher, S. O., Patel, R. M., Landry, J. A., Bonnell, R. B. ve Viau, A. A. (2003). Classification of hyperspectral data by decision trees and artificial neural networks to identify weed stress and nitrogen status of corn. Comput. Electron. Agricult., 39(2), 67-93.

Gualtieri, J. A. ve Cromp, R. F. (1999). Support vector machines for hyperspectral remote sensing classification. Proc. SPIE içinde (C. 3584, ss. 221-232).

Ham, J., Chen, Y., Crawford, M. M. ve Ghosh, J. (2005). Investigation of the random forest framework for classification of hyperspectral data. IEEE, 43(3), 492-501.

Keshava, N. (2004). Distance metrics and band selection in hyperspectral processing with applications to material identification and spectral libraries. IEEE Transactions on Geoscience and Remote Sensing, 42(7), 1552-1565.

Li, J., Bioucas-Dias, J. M. ve Plaza, A. (2012). Spectral-Spatial Hyperspectral Image Segmentation Using Subspace Multinomial Logistic Regression and Markov Random Fields. IEEE, 50(3), 809-823.

Li, J., Bioucas-Dias, J. M. ve Plaza, A. (2013). Semisupervised Hyperspectral Image Classification Using Soft Sparse Multinomial Logistic Regression. IEEE, 10(2), 318-322.

Li, W. ve Du, Q. (2014). Joint Within-Class Collaborative Representation for Hyperspectral Image Classification. IEEE, 7(6), 2200-2208.

Ma, L., Crawford, M. M. ve Tian, J. (2010). Local Manifold Learning-Based $k$-NearestNeighbor for Hyperspectral Image Classification. IEEE, 48(11), 4099-4109.

Manolakis, D. ve Shaw, G. (2002). Detection algorithms for hyperspectral imaging applications. IEEE, 19(1), 29-43.

Melgani, F. ve Bruzzone, L. (2004). Classification of hyperspectral remote sensing images with support vector machines. IEEE Transactions on Geoscience and Remote Sensing, 42(8), 1778-1790.
Ratle, F., Camps-Valls, G. ve Weston, J. (2010). Semisupervised Neural Networks for Efficient Hyperspectral Image Classification. IEEE, 48(5), 2271-2282.

Shukla, A. ve Kot, R. (2016). An Overview of Hyperspectral Remote Sensing and its applications in various Disciplines. IRA-Int. J. Appl. Sci., 5(2), 85-90.

Stavrakoudis, D. G., Galidaki, G. N., Gitas, I. Z. ve Theocharis, J. B. (2012). A Genetic FuzzyRule-Based Classifier for Land Cover Classification From Hyperspectral Imagery. IEEE, 50(1), 130-148.

Sun, X., Qu, Q., Nasrabadi, N. M. ve Tran, T. D. (2014). Structured Priors for SparseRepresentation-Based Hyperspectral Image Classification. IEEE, 11(7), 1235-1239.

Tropp, J. A. ve Gilbert, A. C. (2007). Signal Recovery From Random Measurements Via Orthogonal Matching Pursuit. IEEE Transactions on Information Theory, 53(12), 4655-4666.

Tu, B., Zhang, X., Kang, X., Zhang, G., Wang, J. ve $\mathrm{Wu}$, J. (2018). Hyperspectral Image Classification via Fusing Correlation Coefficient and Joint Sparse Representation. IEEE Geoscience and Remote Sensing Letters, 15(3), 340-344.

Yan, L., Cui, M. ve Prasad, S. (2018). Joint Euclidean and Angular Distance-Based Embeddings for Multisource Image Analysis. IEEE Geoscience and Remote Sensing Letters, 15(7), 1110-1114.

Zhang, H., Li, J., Huang, Y. ve Zhang, L. (2014). A Nonlocal Weighted Joint Sparse Representation Classification Method for Hyperspectral Imagery. IEEE Journal of Selected Topics in Applied Earth Observations and Remote Sensing, 7(6), 2056-2065.

Zhong, Y. ve Zhang, L. (2012). An Adaptive Artificial Immune Network for Supervised Classification of Multi-/Hyperspectral Remote Sensing Imagery. IEEE, 50(3), 894-909. 